\title{
Similar Tensor Arrays \\ - A Framework for Storage of Tensor Array Data
}

\author{
Anders Brun ${ }^{1,2,3}$, Marcos Martin-Fernandez ${ }^{4}$, Burak Acar ${ }^{5}$, \\ Emma Munoz-Moreno ${ }^{4}$, Leila Cammoun ${ }^{6}$, Andreas Sigfridsson ${ }^{2,3,7}$, \\ Dario Sosa-Cabrera $^{8}$, Björn Svensson ${ }^{2,3}$, Magnus Herberthson ${ }^{9}$, and \\ Hans Knutsson ${ }^{2,3}$
}

\begin{abstract}
This chapter describes a framework for storage of tensor array data, useful to describe regularly sampled tensor fields. The main component of the framework, called Similar Tensor Array Core (STAC), is the result of a collaboration between research groups within the SIMILAR network of excellence. It aims to capture the essence of regularly sampled tensor fields using a minimal set of attributes and can therefore be used as a "greatest common divisor" and interface between tensor array processing algorithms. This is potentially useful in applied fields like medical image analysis, in particular in Diffusion Tensor MRI, where misinterpretation of tensor array data is a common source of errors. By promoting a strictly geometric perspective on tensor arrays, with a close resemblance to the terminology used in differential geometry, STAC removes ambiguities and guides the user to define all necessary information. In contrast to existing tensor array file formats, it is minimalistic and based on an intrinsic and geometric interpretation of the array itself, without references to other coordinate systems.
\end{abstract}

\footnotetext{
${ }^{1}$ Centre for Image Analysis, Box 337, SE-751 05, SLU, Uppsala, Sweden.

anders@cb.uu.se (corresponding author) . 2 Division of Medical Informatics, Department of Biomedical Engineering, Linköping University, Linköping, Sweden. \{bjosv, knutte\}@imt.liu.se. 3 Center for Medical Image science and Visualization (CMIV), Linköping University, Linköping, Sweden. . ${ }^{4}$ Laboratorio de Procesado de Imagen (LPI), Dept. Teoría de la Señal y Comunicaciones e Ingeniería Telemática, Universidad de Valladolid, Spain.

marcma@tel.uva.es, emunmor@lpi.tel.uva.es. 5 Electrical \& Electronics Engineering Department, Boğaziçi University, Istanbul, Turkey. acarbu@boun.edu.tr . 6 Signal Processing Institute (ITS), Ecole Polytechnique Fédérale Lausanne (EPFL), Lausanne, Switzerland. leila.cammoun@epfl.ch . Division of Clinical Physiology, Department of Medicine and Care, Linköping University, Linköping, Sweden. sigge@imv. liu . se ${ }^{7}$ Center for Technology in Medicine, Dept. Señales y Comunicaciones, University of Las Palmas de Gran Canaria, Spain. dario@ctm.ulpgc.es ${ }^{8}$ Department of Mathematics, Linköping University, Linköping, Sweden. maher@mai.liu.se
} 


\section{Introduction}

Trying to describe facts of the world, facts of worlds that may only exists in our imagination, or regularly sampled tensor fields, it is appropriate to start with a quote:

"Wovon man nicht sprechen kann, darüber muss man schweigen."1

- Ludwig Wittgenstein, from Tractatus Logico-Philosophicus [19]

In this chapter, we define a minimalistic tensor array file format called Similar Tensor Array Core (STAC). It is designed to capture the essence of tensor arrays and it makes no effort to describe anything else. If something is not a regularly sampled tensor field, it cannot be described by STAC. It is also with a reference to the late thinking of Wittgenstein that we now write this chapter. We define a canonical standard for storage of tensor arrays, to be used as such. However, we also define this standard to curiously see what response such an action will trigger in on the tensor image processing community.

Tensors and tensor fields are basic tools in differential geometry and physics, to describe geometric and physical quantities that remain invariant under coordinate transformations. Examples include mathematics and physics in general [10], continuum mechanics [8], general relativity [16], diffusion in the human body [17], and local image features in 2-D and higher dimensional images [1]. In computer programs, tensors and tensor fields are often implemented using arrays, with indices corresponding to both spatial dimensions (e.g. $x, y$ and $z$ ) and tensor indices (e.g. $i$ and $j$ ). Due to the lack of support for tensors in most programming languages, programmers have themselves come up with different conventions for storing tensor data. One of the things that has caused confusion in Diffusion Tensor MRI applications is the relation between the basis vectors of the tensors and the basis vectors of the space where the tensors have been sampled. Perhaps due to a limited view of diffusion tensors, that they are simply $3 \times 3$ matrices, the geometric nature of tensors has sometimes been overlooked and lost. For this reason, we propose a standard for storage of tensor arrays, to promote a geometric view of tensors in the image processing community and make it easier to exchange data between computer programs and between researchers.

We propose a compact file format that is able to store regularly sampled tensor fields, in arbitrary dimensions. The approach is minimalistic, canonical and geometric. It can store tensor arrays, and nothing but tensor arrays. There is a unique encoding of a particular tensor array and the tensor components are strictly coupled to the geometric arrangement of the array of samples. It aims to capture the very essence of tensor fields, using arrays, in a way that is compatible with mathematics, physics and computer programming. We have divided the work on Similar Tensor Arrays into two parts:

- Similar Tensor Array Core (STAC). This is the basic data type for storage of tensor arrays. It promotes simplicity and safety in processing, communication and storage of regularly sampled tensor field data.

1 "Whereof one cannot speak, thereof one must be silent." 